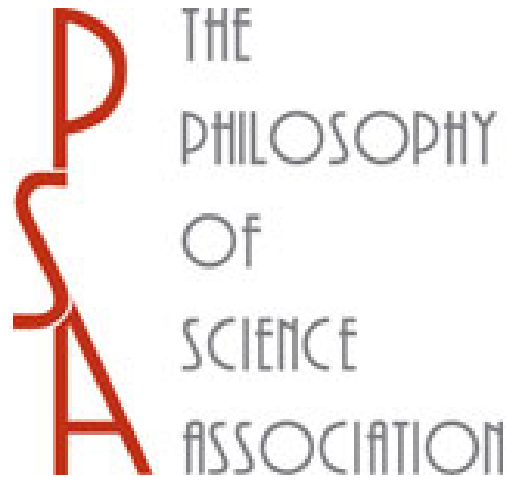

Conservation Laws in Scientific Explanations: Constraints or Coincidences?

Author(s): Marc Lange

Source: Philosophy of Science, Vol. 78, No. 3 (July 〈year>2011</year〉), pp. 333-352

Published by: The University of Chicago Press on behalf of the Philosophy of Science Association

Stable URL: http://www.jstor.org/stable/10.1086/660299

Accessed: 06/07/2011 10:47

Your use of the JSTOR archive indicates your acceptance of JSTOR's Terms and Conditions of Use, available at http://www.jstor.org/page/info/about/policies/terms.jsp. JSTOR's Terms and Conditions of Use provides, in part, that unless you have obtained prior permission, you may not download an entire issue of a journal or multiple copies of articles, and you may use content in the JSTOR archive only for your personal, non-commercial use.

Please contact the publisher regarding any further use of this work. Publisher contact information may be obtained at http://www.jstor.org/action/showPublisher?publisherCode=ucpress.

Each copy of any part of a JSTOR transmission must contain the same copyright notice that appears on the screen or printed page of such transmission.

JSTOR is a not-for-profit service that helps scholars, researchers, and students discover, use, and build upon a wide range of content in a trusted digital archive. We use information technology and tools to increase productivity and facilitate new forms of scholarship. For more information about JSTOR, please contact support@jstor.org. 


\title{
Conservation Laws in Scientific Explanations: Constraints or Coincidences?*
}

\author{
Marc Lange ${ }^{\dagger}$
}

A conservation law in physics can be either a constraint on the kinds of interaction there could be or a coincidence of the kinds of interactions there actually are. This is an important, unjustly neglected distinction. Only if a conservation law constrains the possible kinds of interaction can a derivation from it constitute a scientific explanation despite failing to describe the causal/mechanical details behind the result derived. This conception of the relation between "bottom-up" scientific explanations and one kind of "top-down" scientific explanation is motivated by several examples from classical and modern physics.

1. Introduction. At the close of "Four Decades of Scientific Explanation," Wesley Salmon (1989, 182-85) contrasts "bottom-up" and "top-down" approaches to scientific explanation (borrowing this terminology from Philip Kitcher). An explanation taking a bottom-up approach describes the causal processes, interactions, and (often hidden) mechanisms responsible for particular occurrences or general regularities, as when the kinetic-molecular theory of gases explains Boyle's law. In contrast, an explanation taking a top-down approach subsumes the explanandum under some extremely general principles, thereby unifying it with other facts - as when general relativity's principle of equivalence explains the behavior of a helium-filled balloon in an airplane accelerating for takeoff. (A bottom-up explanation might instead cite the cabin air pressure gradient.) Salmon suggests that there exist both top-down and bottom-up

* Received July 2010; revised October 2010.

$\dagger$ To contact the author, please write to: Department of Philosophy, University of North Carolina at Chapel Hill, CB 3125, Chapel Hill, NC 27599-3125; e-mail: mlange@email .unc.edu.

Philosophy of Science, 78 (July 2011) pp. 333-352. 0031-8248/2011/7803-0001\$10.00

Copyright 2011 by the Philosophy of Science Association. All rights reserved. 
scientific explanations. They can provide different kinds of understanding of the same fact: "It is my present conviction that both of these explanations are legitimate and each is illuminating in its own way" (183-84).

Salmon's view may seem an uneasy rapprochement between causal/ mechanical and unificationist pictures of scientific explanation. Suppose we recognize some detailed causal/mechanical account as specifying how a given explanandum comes about. Then we may well find it difficult to see how a top-down account could possibly make any explanatory contribution at all. Of course, a top-down argument might have allowed us to discover the explanandum without having to rely on any theory of the microphysical details responsible for it. But this valuable heuristic role should not be confused with an explanation. Although Salmon does not mention it, Einstein (1954) famously draws a similar distinction between bottom-up "constructive theories" (Einstein's example is the same as Salmon's: the kinetic-molecular theory of gases) and top-down "theories of principle" (Einstein's examples: thermodynamics and relativity). But in contrast to Salmon, Einstein is generally interpreted as regarding only constructive theories as explanatory since he says, "When we say that we have succeeded in understanding a group of natural processes, we invariably mean that a constructive theory has been found which covers the processes in question" (228). Many philosophers (e.g., Brown 2005; DiSalle 2006, 115-16) have agreed with Einstein while so interpreting him, and many philosophers (e.g., Elster 2007) regard all explanations as causal explanations. Bottom-up explanations can thus make it difficult to acknowledge top-down explanations. The reverse can also happen. Suppose we see how an outcome follows from some general principle that must hold for any possible mechanism. Then, although an account of the particular mechanism actually at work supplies us with further information about the case, we may well regard this welter of causal detail as explanatorily superfluous since the result would still have obtained on any mechanism obeying the general principle.

Nevertheless, I believe that Salmon is correct in contending that both bottom-up and top-down arguments can be explanatory. One important kind of top-down argument in physics proceeds from conservation laws. Yet when some relationship can receive a bottom-up, causal/mechanical explanation, it may well be difficult to see how its derivation from a conservation law could explain it rather than merely be a convenient means of predicting it. In this article, I will first sharpen this challenge to the explanatory power of conservation laws and then show how this challenge can be met. I will identify what it would take for an argument from conservation laws to explain despite failing to describe the underlying causal mechanism. I will identify a distinctive explanatory contribution that conservation laws can make - a contribution that cannot be made 


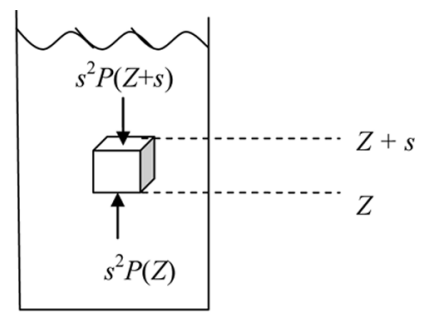

Figure 1.

by any causal/mechanical account (no matter how detailed) of the same explanandum. In particular, I will argue that a conservation law explains when it is a constraint on the kinds of interaction there could have been and not when it is a coincidence of the kinds of interactions there happen to be. I will elaborate this modal distinction and show its importance to scientific explanation in several examples from classical and modern physics.

2. A Challenge to Explanations Appealing to Conservation Laws. Let's take a simple example of a putative explanation using a conservation law - in this case, energy conservation - and let's compare this "explanation" to the standard causal/mechanical explanation of the same fact. (For simplicity, I assume classical physics throughout.) The explanandum is Archimedes' Principle: that the buoyant force on a body surrounded by an ideally incompressible, nonviscous fluid in a container at rest in a uniform downward gravitational field equals the weight of the fluid displaced by the body. The bottom-up explanation of Archimedes' Principle is hydrostatic. As the physicist James Trefil says, "Archimedes' principle can be understood in terms of kinetic theory. . . . For a completely submerged object, the pressure will be less on its top than on its bottomthe molecules of the fluid will be hitting the bottom of the object with a greater force than those hitting the top. This is the molecular origin of the upward buoyant force" (2003, 22).

More fully (see, e.g., Pnueli and Gutfinger 1992), the hydrostatic explanation begins by defining a fluid as a continuum that cannot resist a change in its shape, so the force that one fluid parcel feels from contact with another is perpendicular to its surface. Since the fluid parcels are all at equilibrium (i.e., not accelerating), it follows (from Newton's second law of motion) that the forces exerted on all sides of a fluid parcel sum to zero. Shrinking the parcel to a point, we find that at any point in the fluid, there is a well-defined pressure $P$ that is equal in all directions.

Take a cubical parcel of fluid (see fig. 1), each side of length $s$, its faces 


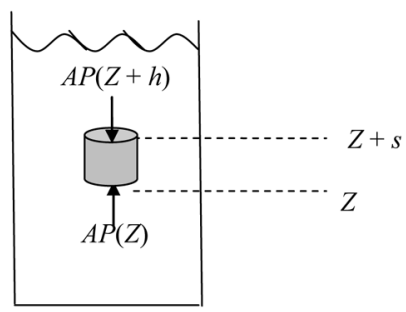

Figure 2.

aligned with the coordinate axes and small enough that the pressure is uniform over any given side. Since the pressure force on a face is perpendicular to it, the total force in the $z$ (upward) direction exerted on the parcel by contact with the surrounding fluid is the magnitude $s^{2} P(Z)$ of the force on the cube's base, all points of which have $Z$ as their $z$-coordinate, minus the magnitude $s^{2} P(Z+s)$ of the force on the cube's top, totaling $-s^{3}[P(Z+s)-P(Z)] / s$. Gravity is the only force on the parcel that does not act by contact; it has only a $z$-component: $-\rho s^{3} g$, where $\rho$ is the fluid's density. By Newton's second law, $\rho s^{3} g-s^{3}[P(Z+s)-P(Z)] / s=m a_{z}$ for the parcel's mass $m$ and $z$-acceleration $a_{z}$. Dividing by $s^{3}$ and taking the limit as $s$ approaches zero, we find $-\rho g-(d P / d z)(Z)=\rho a_{z}$. Since the fluid's parcels are at equilibrium, $a_{z}=0$, so $(d P / d z)(Z)=-\rho g$.

Suppose (for simplicity) that the submerged body is a cylinder of height $h$ with horizontal top and bottom surfaces, each of area $A$ (see fig. 2). Let $Z$ be the bottom surface's $z$-coordinate. The force downward on the top surface exerted by the fluid above it at pressure $P(Z+h)$ is $A P(Z+$ $h$ ); the upward force on the cylinder's bottom surface exerted by the fluid below it at pressure $P(Z)$ is $A P(Z)$. (The pressure exerts forces only perpendicular to the cylinder's surface, so no upward or downward force is exerted on the cylinder's sides.) The net $z$-force is then $A[P(Z)-P(Z+$ $h)]=-A \int_{Z}^{Z+h}(d P / d z) d z=A \rho g h$. The cylinder's volume is $A h$, so the mass of the fluid it displaces is $A \rho h$. That fluid's weight is $A \rho g h$, which (we just saw) equals the buoyant force. ${ }^{1}$

That was a bottom-up explanation of Archimedes' Principle. A topdown derivation from energy conservation is sometimes presented as also explaining why the principle holds, as in a physics journal article (Leroy

1. For an arbitrarily shaped object, the calculation yields the same result: the body is decomposed into thin cylindrical elements, and although their top and bottom surfaces may tilt arbitrarily relative to the horizontal, the horizontal components of the pressure forces perpendicular to the body's variously tilted surface elements at any given level $z$ add to zero. 


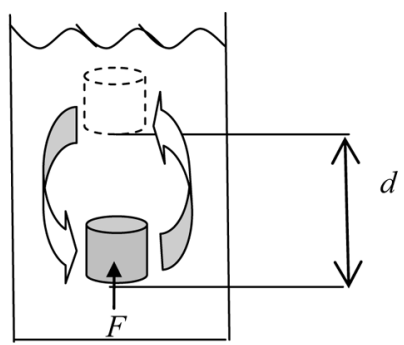

Figure 3.

$1985,56)$ that said that in view of the top-down derivation, "the origin of the buoyancy force need no longer be mysterious and therefore hidden behind a principle; it can be explained in physical terms." (For similar remarks, see also Rudiak 1964; Tarasov and Tarasova 1973, 121; and Keeports 2002.)

Here is the top-down derivation. Suppose an applied upward force $F$ just suffices to raise the submerged body by a vertical distance $d$; the body is moved arbitrarily slowly without any superfluous motion of the fluid (see fig. 3). By energy conservation, the work $F d$ thereby done on the system equals the energy added to the system. Since the fluid is ideal, none of the work goes into overcoming friction (which would have raised the fluid's internal energy), and so it all goes into changing the system's gravitational potential energy. The body's gravitational potential energy rises by $M_{b} g d$, where $M_{b}$ is the body's mass. As the body rises, fluid flows downward to fill the space it vacates. As a result of its journey, the body effectively trades places with a fluid parcel of the body's size and shape that was initially located at a distance $d$ above the body's initial position (see fig. 3). That parcel thus descends by $d$, so its gravitational potential energy diminishes by $M_{p} g d$, where $M_{p}$ is the parcel's mass. Setting the work done equal to the net increase in the system's potential energy, we find $F d=M_{b} g d-M_{p} g d$. Therefore, $F=M_{b} g-M_{p} g$. By contrast, had there been no fluid, then the force just sufficient to raise the body would have been simply $M_{b} g$ - just enough to balance the body's weight. But with the fluid present, we need less force by $M_{p} g$ - the weight of the fluid displaced by the body. That upward force supplementing $F$ is the buoyant force.

Of course, this argument does not reveal how the buoyant force acts. Admittedly, the bottom-up explanation also fails to identify the fundamental force at work in molecular collisions. But it does at least reveal that the buoyant force is exerted through these collisions. The derivation 
from energy conservation shows that the buoyant force must be present but fails to uncover the causal mechanism by which it acts. Thus, a distinctive explanatory contribution is made by the hydrostatic derivation.

However, it would be question begging to argue that the derivation from energy conservation is not explanatory simply because it is not a bottom-up, causal/mechanical account. Accordingly, let's sharpen this challenge to the top-down derivation's explanatory power. Energy conservation's role in this derivation is apparently only to ensure that gravitational interactions conserve energy, thereby making "gravitational potential energy" well defined and allowing the work done on the system to equal the change in its gravitational potential energy. Energy conservation as a comprehensive principle - as covering all fundamental types of interaction - appears to be largely irrelevant. That is, if we think of energy conservation as a long conjunction (that gravitational interactions conserve energy and electrical interactions conserve energy and magnetic interactions conserve energy, and so on, and there are no other kinds of interactions) with one conjunct for each species of fundamental interaction, then most of these conjuncts are irrelevant to the top-down "explanation" of Archimedes' Principle. ${ }^{2}$

Of course, their irrelevance does not demonstrate the irrelevance of the fact that gravitational interactions conserve energy. But if they are irrelevant to explaining Archimedes' Principle, then the comprehensive law of energy conservation is also irrelevant since it includes irrelevant conjuncts. Hence, each separate top-down "explanation" that apparently appeals to the comprehensive law of energy conservation in order to explain some or another phenomenon actually uses only the fact that energy is conserved by the particular types of interaction at work in producing that phenomenon. Therefore, these derivations do not all proceed from a common premise (the comprehensive law of energy conservation). Apparently, then, these various derivations fail to achieve the kind of grand unification that was supposed (by Salmon, at least) to be the source of their explanatory power. A top-down argument, then, does not explain the buoyant force, although it still allows us to calculate the force's strength without having to deal with the complicated causal/mechanical details we encountered in proceeding from the bottom up.

We have, here, a challenge to top-down explanations that appeal to conservation laws. To see what we should make of this challenge, let's identify some of its presuppositions and evaluate their plausibility.

Let's first examine the challenge's final step: that since the derivation

2. By "irrelevant," I mean explanatorily irrelevant, not irrelevant confirmation-wise. That various species of interaction all conserve energy might be some evidence that a given other species does too. 
of Archimedes' Principle from energy's conservation in gravitational interactions cannot unify Archimedes' Principle with the results of nongravitational interactions, the derivation cannot achieve the kind of grand unification that would be needed to supply it with explanatory power, and so the derivation cannot explain why Archimedes' Principle holds. This step presupposes that a top-down explanation must acquire its explanatory power from its capacity to unify. However, the challenge to the explanatory power of the comprehensive principle of energy conservation can actually do without this unificationist conception of the source of explanatory power. To launch the challenge, it is enough that energy conservation cannot explain if it fails to unify across the range of different kinds of interactions; the challenge need not presuppose that any explanatory power possessed by the conservation law must arise from some sort of unification it achieves. The unification may be a product of the explanation.

Plausibly, energy conservation explains only if it unifies various phenomena involving different kinds of interactions. Precisely this sort of unification is frequently emphasized as the most notable achievement of the conservation laws. As Richard Feynman $(1967,59)$ puts it, "When learning about the laws of physics you find that there are a large number of complicated and detailed laws, laws of gravitation, of electricity and magnetism, nuclear interactions, and so on, but across the variety of these detailed laws there sweep great general principles which all the laws seem to follow. Examples of these are the principles of conservation." What sort of unification would a grand conservation law thus produce? It would unify by being an important premise that is shared by explanations of various phenomena resulting from otherwise unrelated kinds of interactions. That is, it would be an important premise common to various derivations (such as the derivation of Archimedes' Principle) that explain particular facts having bottom-up explanations involving entirely distinct kinds of forces. As a common explainer, it would then unify in much the same way as a common cause does (although, of course, no law is, strictly speaking, a cause).

But this "unification" would be trivial if a suitable common premise could be produced on the cheap: simply by conjoining premises appearing in separate explanations. For example, consider an explanation of the gravitational force between a given pair of bodies that appeals to Newton's gravitational-force law, and also consider a Coulomb's-law explanation of the electrostatic force between a particular pair of bodies. We cannot transform these two explanations into explanations that share an important premise (constituting a common explainer) merely by replacing both of the force laws in the respective explanations with their conjunction. The two derivations would then still be valid, but having this arbitrary 
conjunction as a premise would deprive each of them of any explanatory power. That energy conservation might be a similar arbitrary conjunction (of the fact that gravitational interactions conserve energy and electrical interactions conserve energy and so on) was the core of the challenge I posed to the conservation law's explanatory power.

If energy conservation fails to unify across the various actual kinds of interaction, couldn't Archimedes' Principle still be explained by energy's conservation in gravitational interactions? Perhaps it could. But suppose that this derivation of Archimedes' Principle invokes energy conservation merely as holding of gravitational interactions; suppose the comprehensive principle of energy conservation is an arbitrary conjunction since each conjunct is explained by a different force law, and so the principle is explanatorily irrelevant to Archimedes' Principle because many conjuncts are explanatorily irrelevant. (More on this in a moment.) Then energy's conservation in gravitational interactions is explained by the gravitationalforce law, and so an appeal to gravity's conserving energy becomes just an appeal to an important feature of that force law. The derivation of Archimedes' Principle from energy conservation in gravitational interactions, then, appears not to be a top-down explanation. Rather, the gravitational-force law helps to explain both Archimedes' Principle and the fact that gravitational interactions conserve energy - so that even if the derivation of Archimedes' Principle from gravity's conserving energy is an explanation (rather than merely a means of calculating the buoyant force's strength without having to deal with the intricacies of the hydrodynamic derivation), it is just another bottom-up explanation. Energy's conservation in gravitational interactions, then, does not help to explain anything about the gravitational-force law that powers the hydrodynamic explanation. On the contrary, the force law helps to explain why energy is conserved in gravitational interactions. Energy conservation does not stand "above" the force laws at all.

To identify what it would amount to for energy conservation to stand above the force laws, properly positioned to figure in top-down explanations, is exactly the challenge I issued earlier. Let's now look more closely at another part of that challenge: that the comprehensive law of energy conservation is an "arbitrary conjunction" of separate conservation laws concerning various distinct kinds of interactions rather than (as Feynman says) a single principle sweeping across the various force laws. In that case, I suggested, Archimedes' Principle is not explained by the comprehensive conservation law because that law includes explanatorily irrelevant conjuncts. Of course, that these conjuncts are unneeded for entailing Archimedes' Principle fails to show that they do not help to explain Archimedes' Principle. That the scope of some law serving as a premise in an explanation can be diminished without rendering the der- 
ivation invalid does not show that the derivation appeals to some explanatorily irrelevant premises and so fails to explain. For example, the 4-dyne electrostatic force between a given pair of point charges at rest is explained by Coulomb's law (and the fact that the two charges are 1 centimeter apart and two statcoulombs each), even though the derivation remains valid if Coulomb's law is replaced by a much narrower Coulombic law: that any two point charges of 2 statcoulombs each, at rest 1 centimeter apart, exert 4-dyne electrostatic forces on each other. Coulomb's law is not explanatorily irrelevant to the 4-dyne force, even though "most" of Coulomb's law can be omitted from the derivation without undermining its validity.

In contrast, however, suppose we take the original explanation and replace Coulomb's law with that law conjoined with some other arbitrary law, such as Newton's gravitational-force law. Then we no longer have an explanation of the 4-dyne electrostatic force. ${ }^{3}$ With an arbitrary conjunct, the derivation remains deductively valid but contains explanatorily irrelevant premises. The challenge, then, is to specify what it would take for the comprehensive law of energy conservation to be like Coulomb's law (which is not an arbitrary conjunction) rather than like the (arbitrary) conjunction of Coulomb's law with Newton's gravitational-force law.

There are actually two challenges here. One challenge is to understand what makes Coulomb's law different from the conjunction of Coulomb's law with the gravitational-force law-that is, what makes the latter but not the former an "arbitrary conjunction." Here is perhaps another way to express this challenge: What makes the electrostatic force constitute a single natural kind of interaction (or, at least, a subclass of a single natural kind), and, in contrast, what makes gravitational and electrostatic forces not belong to a single natural kind of force? (I shall later sketch a possible answer to this question.) The second challenge (on which I shall mainly

3. Perhaps it initially seems to you that the addition of Newton's law does not ruin the explanation. I insist that it does: gravitational forces play no part in generating the force to be explained, so the gravitational-force law is explanatorily irrelevant, and an explanation can include nothing explanatorily irrelevant. Any temptation to think that the gravitational-force law does not ruin the explanation, but merely constitutes a harmless superfluous premise within an explanation, may be accounted for by the fact that it is easy to see how one could start with the derivation using Coulomb's law conjoined with the gravitational-force law and then transform it into an explanation (simply by slicing off the gravitational-force law). Therefore, the derivation from the arbitrary conjunction "contains" an explanation and can inform us of an explanation without itself constituting an explanation. Compare this example involving Coulomb's law conjoined with the gravitational-force law to the example in the "notorious" footnote 33 (originally n. 28) in Hempel and Oppenheim's landmark 1948 essay on explanation (Hempel 1965, 273), where the conjunction of Kepler's and Boyle's laws is described as entailing but not explaining Kepler's laws. 
focus) is to understand what it would take for the comprehensive law of energy conservation not to be an arbitrary conjunction of various conservation laws regarding separate kinds of force. That is, what would enable the comprehensive law of energy conservation to help explain Archimedes' Principle despite the derivation's requiring for its validity only energy's conservation by a single kind of force?

This second challenge is made especially serious by the fact that the comprehensive law of energy conservation seems a good deal like the conjunction of Coulomb's law with the gravitational-force law. In other words, it seems to be the conjunction of several principles concerning distinct natural kinds of force (namely, that gravitational interactions conserve energy and electrical interactions conserve energy and so on). Presumably, whatever it is that would make the comprehensive law of energy conservation able to explain Archimedes' Principle would also enable such a top-down explanation to contribute something that cannot be supplied by the bottom-up, hydrodynamic explanation of Archimedes' Principle. I shall now try to identify what that contribution would beand to use it to suggest what it would take for the comprehensive law of energy conservation to explain Archimedes' Principle.

\section{Conservation Laws as Constraints or Coincidences.}

3.1. Introducing the Distinction between Constraints and Coincidences. As I noted, the hydrostatic explanation must presuppose that an ideal fluid left to itself in a container at rest in a uniform gravitational field is at equilibrium; that is, its parcels are not accelerating. This fact about ideal fluids could apparently be explained by a complete inventory of the actual kinds of forces: none disturbs such a fluid's equilibrium.

Newton's second law of motion does not preclude the existence of additional kinds of forces on the fluid. Newton's law requires only that any such forces be associated with corresponding accelerations. Suppose there had been an additional force that any fluid, left to itself, naturally experiences - a force not among the actual kinds. In particular, suppose that there had been a further fundamental force law requiring that a fluid's parcels, initially at rest, feel a force that puts them into a top-to-bottomto-top circulation: without the intervention of any outside agency, some higher parcels are continually pulled downward and lower parcels pushed upward. The details of the parcels' resulting acceleration do not matter. The point is that such a force would not conflict with Newton's second law of motion, but it would interfere with the hydrostatic explanation of Archimedes' Principle. Therefore, the premises used by that explanation must rule out such a force by entailing that the fluid, left to itself, is in equilibrium. 
As far as the hydrostatic explanation is concerned, the fact that no actual kind of force produces such a circulation could be just a coincidence. In other words, the reason why no actual kind of force produces such a circulation could be that one type of force does not, another type does not, and so forth, for every actual type of force, with each type of force having this feature for its own separate reason (namely, because of its own force law). That two actual types of fundamental force (e.g., electric and gravitational) are alike in failing to induce a circulation would then have no common explanation. The lack of any common explanation would make it a coincidence that the two forces are alike in failing to induce a circulation - just as if you go to the mall this afternoon for one reason and I go for an unrelated reason, then our both being there at the same time is a coincidence since my being there and your being there have no common explainer. ${ }^{4}$ The hydrostatic explanation offers no common explanation of the various actual types of forces all failing to induce a circulation since the hydrostatic explanation simply starts with the particular kinds of forces there actually are. (Every explanation must start somewhere.)

In contrast, I will now suggest, a top-down explanation employs the law of energy conservation as a constraint on the kinds of forces there could have been. A force arising from no outside agency that would make the fluid parcels spontaneously begin to circulate from rest would violate energy conservation: in beginning to circulate, the parcels' kinetic energy would increase, but their total potential energy would be unchanged. (As ascending parcels gain gravitational potential energy, descending parcels lose an equal quantity of it.) Energy conservation as a constraint rules out any circulating force and so constitutes a common explainer of the fact that (for instance) electric and gravitational forces are alike in failing to induce fluid circulation. ${ }^{5}$

A top-down explanation of Archimedes' Principle from the comprehensive law of energy conservation would thus make an explanatory contribution that cannot be made by a bottom-up explanation, no matter how detailed. (I will say more about this contribution in a moment.) But

4. Or, at least, they have no common explainer of any interest in the context of some particular demand for an explanation. Perhaps we both traveled to the mall by using our own cars, so the principles of the internal combustion engine are common explainers of our each arriving at the mall. But these are not the sorts of explainers that we would typically be asking for.

5. The points I make in terms of Archimedes' Principle could instead have been made by using Stevinus's 1586 clootcrans argument for the law of the inclined plane; energy conservation would then preclude the chain loop, having been laid at rest across the prism, from beginning to turn. Halonen and Hintikka $(1999,32)$ deem this derivation explanatory. My aim is to determine what would make it so. 
for energy conservation to supply this explanation, the conservation law cannot be a coincidence. In other words, it cannot be that the various force laws explain why energy is always conserved. Rather, that one force conserves energy and that another does, too, must have a common explanation in the conservation law. For the law of energy conservation to explain why no circulation-inducing force exists, the law has to be a constraint on the fundamental forces there could be; it has to demand that whatever the fundamental forces happen to be, they conserve energy. If it constrains the forces there could be, then the conservation law is not equivalent to the long conjunction from section 2: that gravitational interactions conserve energy and electrical interactions conserve energy and magnetic interactions conserve energy, and so on, and there are no other kinds of interactions. The conservation law does not refer to particular kinds of interactions or depend on the kinds of interactions there happen to be. It is not an arbitrary conjunction.

Earlier, we saw Feynman describe energy conservation as a constraint: that the conservation laws are general principles that all of the force laws must follow. Likewise, Mark Steiner $(1978,22)$ writes, "Laws of conservation are simply not causal laws. They provide constraints on what is allowed to happen." I am not arguing that conservation laws do in fact constitute such constraints. My aim is merely to identify what it would take for an argument from the conservation laws to explain if its explanatory power does not derive from its describing the underlying causal mechanism - that is, what it would take for such an argument to constitute a top-down explanation.

A bottom-up explanation of Archimedes' Principle takes for granted the fluid's equilibrium (or the inventory of fundamental forces), whereas energy conservation explains why the fluid is at equilibrium by making any forces that fail to conserve energy impossible - but only if energy conservation is a constraint on the forces. In that case, the top-down argument makes a distinctive explanatory contribution. Of course, even the bottom-up explanation deems it no accident (i.e., deems it physically necessary) that there exists no circulation-inducing force since the natural laws suffice to fix the inventory of forces. But that every actual type of force is alike in failing to induce fluid circulation is still a coincidence if there is no common explanation for two different types of force being alike in this respect. By contrast, it is no coincidence if energy conservation constrains the possible force laws.

3.2. The Distinction Defined in Terms of Counterfactuals. Let's now try to be more precise about what it would take to make the conservation law a constraint rather than a coincidence. To say that energy conservation constrains the possible force laws is to say that energy would still have 
been conserved, even if there had been additional kinds of force (i.e., forces that are not electric or gravitational, etc.) acting together with the actual kinds. On the other hand, to say that energy conservation is a coincidence of the actual force laws is to say that it is not the case that energy would still have been conserved, had there been additional kinds of force. Rather, energy is conserved because, as it happens, each of the actual kinds of force conserves energy as a result of its own particular force law. So had there been additional kinds of force, energy might still have been conserved, but then again, it might not have been, depending on the force laws of the additional forces.

One way for a conservation law to be a constraint is for it to follow from other principles that are constraints and so would still have held, even if the there had been additional kinds of force. For instance, as is well known, various classical conservation laws follow from space-time symmetries within a Hamiltonian dynamical framework: energy conservation follows from the laws' invariance under arbitrary temporal displacement, linear momentum conservation from their invariance under arbitrary spatial displacement, and so forth. If the space-time symmetries and dynamical framework would have been no different, even if there had been an additional kind of force, then the conservation laws would still have held, too, and so they constrain the possible force laws. As Eugene Wigner $(1972,13)$ says, "For those [conservation laws] which derive from the geometrical principles of invariance it is clear that their validity transcends that of any special theory - gravitational, electromagnetic, etc.-which are only loosely connected."

In other words, Wigner contends that those symmetries are not coincidences of the particular kinds of forces there happen to be, and so the associated conservation laws transcend the idiosyncrasies of the force laws figuring in bottom-up explanations. (For more on the role of counterfactuals in enabling symmetry principles to explain conservation laws, see Lange [2007, 2009].)

3.3. The Distinctive Explanatory Contribution Made by Constraints. If the comprehensive law of energy conservation constrains the possible force laws, then it explains why no force makes fluid parcels spontaneously begin to circulate from rest, thereby explaining a premise of the hydrodynamic derivation of Archimedes' Principle. Of course, that no actual force would put such a fluid into circulation also follows from an inventory of the various actual kinds of forces. However, that inventory portrays the fact that there is no circulating force as a kind of coincidence; it identifies no common explainer of the fact that electric forces would not put the fluid into circulation and the fact that gravitational forces would not either. In contrast, the absence of a circulating force is no coincidence 
if energy conservation is a constraint on the kinds of forces there could have been. Therefore, if energy conservation is a constraint, then the absence of a circulating force is not explained by the inventory of the kinds of forces there actually are since any such proposed explanation mischaracterizes the explanandum as a coincidental upshot of the particular kinds of forces there happen to be, rather than as necessary in view of the kinds of forces there could have been.

Hence, if energy conservation is a constraint, then the absence of a circulating force has no bottom-up explanation from an inventory of the actual kinds of forces. Rather, it has only a top-down explanation. The same applies to the fact that there is no force that violates energy conservation. If energy conservation is a constraint, then any proposal for explaining why every force conserves energy by appealing to the particular kinds of forces there actually are (i.e., from the bottom up) inaccurately portrays the explanandum as a coincidence. Thus, by making the conservation law no arbitrary conjunction, the conservation law's status as a constraint not only would enable it to withstand the challenge we saw (in sec. 2) to its capacity to explain Archimedes' Principle but also would make a top-down explanation able to contribute something that cannot be supplied by any bottom-up explanation of Archimedes' Principle. (This is exactly the kind of connection we sought at the end of sec. 2.)

3.4. Constraints/Coincidences versus Laws/Accidents. I have cashed out the distinction between energy conservation as a constraint and energy conservation as a coincidence in terms of the conservation law's behavior in connection with counterfactuals. Of course, the distinction between laws and accidents is also frequently cashed out in this way (see Lange 2009). For example, whereas Coulomb's law would still have held, even if there had existed additional charged bodies, the accident that all of the families living on my block have two children might not still have held, had there been additional families living on the block. As a constraint on the force laws, then, a conservation law is in some ways like a law of those laws - a higher-order law. (We saw Feynman describe the conservation laws in similar terms, as "great general principles which all the [force] laws seem to follow.") A standard view is that laws explain their instances, whereas accidents do not (e.g., we cannot explain why my family has two children by appealing to the fact that all of the families on our block have two children since it is just a coincidence that all of those families have two children). Similarly, the fact that various actual forces conserve energy is explained by energy conservation as a constraint on the forces but not by energy conservation as a coincidental similarity among them. If energy conservation is a coincidence, then the fact that 
various actual forces conserve energy is explained by the respective force laws.

However, the distinction I have drawn between constraints and coincidences is not identical to the familiar distinction between laws and accidents. Even as a coincidence rather than a constraint, energy's conservation would logically follow from the laws alone and so would not be an accident. It would be physically necessary. Accordingly, there is an important difference between the counterfactuals used to illustrate the law/accident distinction and the counterfactuals used to illustrate the constraint/coincidence distinction. The counterfactuals used to illustrate the distinction between laws and accidents have antecedents that all represent physical possibilities, such as "had there been additional charged bodies" or "had there been additional families living on our block." In contrast, a physical impossibility is posited by the counterfactual antecedent ("had there been additional kinds of forces") figuring in the distinction between constraints and coincidences; that counterfactual is a counterlegal. Thus, although the distinction between constraints and coincidences is a modal distinction, like the distinction between laws and accidents, the two distinctions are not the same. ${ }^{6}$

\subsection{One Constraint Distinguished from Several. Just as Coulomb's law} would still have held if there had been additional bodies, so likewise an arbitrary conjunction of several laws (such as Coulomb's law conjoined with the gravitational-force law) would still have held had there been additional bodies since each of its conjuncts would still have held. The distinction between Coulomb's law and Coulomb's law conjoined with the gravitational-force law is not the distinction between a law and an accident. Rather, it is the distinction between a single law and an arbitrary conjunction of two laws. An analogous distinction at the level of constraints would be between a single constraint and an arbitrary conjunction of two constraints. (That arbitrary conjunction is not what I have termed a "coincidence" since both conjuncts would still have held, even if there had been additional kinds of forces.) Perhaps these distinctions, too, can be cashed out somehow in terms of differences in behavior in connection with counterfactuals. Let me briefly sketch a tentative proposal along

6. The constraint/coincidence distinction is modal. As a coincidence, energy conservation would be no more necessary than the particular force laws there actually happen to be since those laws would be responsible for it. In contrast, as a constraint on the forces there could have been, energy conservation would be more necessary than the actual force laws (as befits a higher-order law); it would still have held, even if the force laws had been different. 
these lines before returning to the distinction between constraints and coincidences.

If Coulomb's law had been violated sometime before today, then with Coulomb's law "out of the way," there would have been nothing to keep Coulomb's law from being violated after today; it might well, then, have been violated. The same applies to other ways of subdividing Coulomb's law into two "parts"; it is not an arbitrary conjunction of two separate laws. In contrast, the conjunction of Coulomb's law with Newton's gravitational-force law behaves differently: had one of these laws not held, the other would still have held. That the various fundamental force laws behave in this way is frequently assumed by philosophers and physicists describing how different the universe would have been if a given force had been a little bit different. For example, it is frequently remarked that had the strong nuclear force been slightly weaker, then it would have been insufficient to bind two protons together into one nucleus. This remark presupposes that had the strong force been slightly weaker, the mutual electromagnetic repulsion of two protons would have been no different. By the same token, had the gravitational force been slightly stronger, then none of the stars in the "main sequence" except the bluest could have formed - but only because the strengths of the other forces would have been no different (Barrow and Tipler 1986, 322-27; Carter 1990, 132-33). Under these counterlegal antecedents, "all other things" are held fixedand the other force laws count as genuinely other things.

The mutual counterfactual independence of the various force laws cashes out Wigner's remark (quoted earlier) that the various force laws are "only loosely connected." Perhaps similar relations of mutual counterfactual independence likewise help to distinguish a single constraint from an arbitrary conjunction of two constraints. Suppose energy conservation is a single constraint (as it would be if it were explained by a single symmetry principle). Then had gravitational forces failed to conserve energy, the constraint would not have obtained, and so with it "out of the way," there would have been nothing to keep other nonconservative forces from existing. In contrast, suppose energy conservation is actually a conjunction of two constraints (perhaps one constraint covering only kinds of forces that can both attract and repel, and another constraint covering only kinds of forces that either exclusively attract or exclusively repel). Then had one of these constraints not obtained, the other would not have been compromised. Whether it is a single constraint or a conjunction of two constraints, though, energy conservation would not be a coincidence of the kinds of forces there happen to be.

3.6. Other Applications. Let me now return to the idea that conservation laws as constraints must be distinguished from conservation laws 
as coincidences - and that a conservation law can supply a top-down explanation only if it is a constraint. These ideas apply to many examples beyond the simple case involving Archimedes' Principle on which I have concentrated so far.

The status of some conservation laws is not yet known, and consequently their explanatory significance remains unresolved. Consider, for instance, the conservation of baryon number in contemporary physics. By energy conservation, an isolated proton can decay only into particles that have less rest mass than it does, and the proton is the lightest baryon (i.e., the lightest particle with nonzero baryon number). The conservation of baryon number thus entails that the proton is stable. Does baryonnumber conservation explain why the proton is stable? The answer remains controversial.

It is no explanation if the conservation of baryon number is a socalled accidental symmetry (a term that was introduced by Steven Weinberg; see Weinberg 1995, 529). An accidental symmetry reflects merely the particular forces in action at lower-energy regimes rather than some deeper "symmetry of the underlying theory" (529). Accordingly, if baryonnumber conservation is an accidental symmetry, then it may not even hold at higher energies (and so the proton may turn out not to be stable but rather to have an extremely long half-life). But even if an accidental symmetry is unbroken, it would still be a coincidence of the particular kinds of interactions written "by hand" into the underlying theory, and so it would fail to explain. (The stability of the lightest baryon would then help to explain why baryon number turns out to be conserved, not vice versa.)

However, baryon-number conservation may instead turn out to be a consequence of a more fundamental symmetry, in which case it would explain the proton's stability. Thus, whereas some physicists cite baryonnumber conservation as explaining why the proton is stable (e.g., Duffin 1980, 82; Davies 1986, 159), other physicists put scare quotes around "explain" (Lederman and Teresi 1993, 303) or say that the jury is still out (Ne'eman and Kirsh 1996, 150-51). My point is that the uncertainty regarding the conservation law's explanatory power is matched by the uncertainty regarding the law's status as a constraint or coincidence. ${ }^{7}$

7. Kosso (2000) also investigates the distinction between fundamental and accidental symmetries (although not in connection with the explanatory power of conservation laws). He says that a symmetry is accidental in virtue of having no relations to other features of nature; whether a symmetry is fundamental or accidental, according to Kosso, is a "holistic" matter, not a matter of the symmetry's modal status or its capacity to play a particular explanatory role. In contrast, I understand accidental symmetries as symmetries that impose no constraints on the allowable interactions in that the symmetries might not still have held had there been different kinds of interactions. 
4. Conclusion. I would like to return to Archimedes' Principle in order to make two concluding points regarding top-down explanations. First, the distinction between constraints and coincidences applies not only to conservation laws but also to other principles used in top-down derivations. For example, Archimedes' Principle is sometimes derived from the Principle of Virtual Work (PVW; as in Mach 1960, 125). The PVW says that a mechanical system acted on by various outside forces is in equilibrium exactly when no total work is done by those forces in any "virtual displacement," that is, hypothetical infinitesimal change in the positions of the system's components in accordance with the constraints on the system. To derive Archimedes' Principle from the PVW, suppose (for the sake of simplicity) that the submerged body is a cube of side $s$ with horizontal top and bottom faces. If its virtual displacement consists of its descent by $d h$, then a body slab of volume $s^{2} d h$ effectively descends by $s$, and a fluid parcel of the same volume ascends by $s$. Gravity performs virtual work $g \rho_{b} s^{3} d h$ on the body and $-g \rho_{f} s^{3} d h$ on the fluid (for body density $\rho_{b}$ and fluid density $\rho_{f}$ ), so by PVW, the system is in equilibrium if $\rho_{b}=\rho_{f}$. Therefore, any submerged body must experience an upward force that would balance its weight if $\rho_{b}=\rho_{f}$ (i.e., if its weight were equal to that of the fluid it displaces) - that is, an upward force equal to the weight of the displaced fluid.

Whether this derivation from the PVW explains Archimedes' Principle is recognized as depending on whether the PVW is a constraint or a coincidence. A common view (Whewell 1874, bk. VI, chap. ii, sec. 4, 333; Lanczos 1986, 77) is that the PVW holds in virtue of the fact that in each elementary kind of mechanical system (e.g., lever, pulley, wheel), the total work done by the forces of constraint under any virtual displacement is zero. But these conjuncts, one for each kind of simple machine, have no common explanation, so the PVW does not unify. Instead, it is a coincidence and hence not explanatory. Accordingly, Whewell says of the PVW that it "serves verbally to conjoin Laws . . . than to exhibit a connexion in them: it is rather a help for the memory than a proof for the reason."

8. The "principle of least [better: stationary] action" has likewise been interpreted sometimes as a constraint but more commonly as a coincidence. For instance, Planck $(1948,48)$ said that it has generally been regarded as a coincidence: "In present-day physics the principle of least action plays a relatively minor role. It does not quite fit into the framework of present theories. Of course, admittedly it is a correct statement; yet usually it serves not as the foundation of the theory, but as a true but dispensable appendix, because present theoretical physics is entirely tailored to the principle of infinitesimal local effects. . . . Physics hence is inclined to view the principle of least action more as a formal and accidental curiosity than as a pillar of physical knowledge." Clearly, "accidental" here does not mean "lacking in physical necessity" but rather means "a coincidence of the laws": a logical consequence of them where there is no 
Finally, there is another important difference between the hydrostatic and energy-conservation explanations of Archimedes' Principle. As we have seen, the hydrostatic explanation determines that the buoyant force equals the weight of the displaced fluid by first calculating the buoyant force hydrostatically, then independently determining the weight of the displaced fluid, and then noting that these coincide. The weight of the displaced fluid does not play any role in the derivation of the buoyant force. The various factors in the weight $A \rho g h$ of the displaced fluid enter the buoyant force's hydrostatic derivation separately: $h$ arises from the pressure difference between the submerged body's top and bottom, $A$ as the area of the surface on which the pressure force acts, and $\rho g$ from the pressure gradient. Fortuitously, it seems, there arise just the factors that together give the weight of the displaced fluid.

In contrast, this relation between the buoyant force and the weight of the displaced fluid is revealed to be no coincidence by the explanation from energy conservation. There, the weight of the displaced fluid functions as a unit throughout since the displaced fluid descends as the submerged body ascends. That the body's size and shape should combine with the fluid's density is no coincidence, considering that both characterize the displaced parcel of fluid, which itself figures in the derivation. Thus, if the derivation from energy conservation is an explanation (because energy conservation is a constraint), then it is able to explain why the buoyant force turns out to equal the weight of the displaced fluid without portraying this fact as arising by algebraic coincidence.

In short, if energy conservation is a constraint, then the explanation from energy conservation reveals the reason why a quantity equal to the weight of the displaced fluid figures in the expression for the buoyant force. This reason is then not revealed by the hydrostatic explanation because that explanation incorrectly depicts it as a coincidence that each of the various factors in the expression for the weight of the displaced fluid appears in the expression for the buoyant force. In fact, it is no coincidence, if the explanation from energy conservation goes through. Rather, each of the factors in the expression for the weight of the displaced fluid appears for the very same reason in the expression for the buoyant force. With the parcel of displaced fluid, they all enter the derivation together.

\section{REFERENCES}

Barrow, John D., and Frank J. Tipler. 1986. The Anthropic Cosmological Principle. Oxford: Clarendon.

Brown, Harvey R. 2005. Physical Relativity. Oxford: Clarendon.

common explanation of all of its instances. However, Planck himself was inclined to think it unlikely that "the dominance of such a simple law could be a mere accident." 
Carter, Brandon. 1990. "Large Number Coincidences and the Anthropic Principle in Cosmology." In Physical Cosmology and Philosophy, ed. John Leslie, 125-33. New York: Macmillan.

Davies, Paul. 1986. The Forces of Nature. Cambridge: Cambridge University Press.

DiSalle, Robert. 2006. Understanding Space-Time. Cambridge: Cambridge University Press.

Duffin, W. J. 1980. Electricity and Magnetism. 3rd ed. London: McGraw-Hill.

Einstein, Albert. 1954. "What Is the Theory of Relativity?" In Ideas and Opinions, 27-32. New York: Bonanza.

Elster, John. 2007. Explaining Social Behavior. Cambridge: Cambridge University Press.

Feynman, Richard. 1967. The Character of Physical Law. Cambridge, MA: MIT Press.

Halonen, Ilpo, and Jaakko Hintikka 1999. "Unification: It's Magnificent but Is It Explanatory?" Synthese 120:27-47.

Hempel, Carl G. 1965. Aspects of Scientific Explanation and Other Essays in the Philosophy of Science. New York: Free Press.

Keeports, David. 2002. "How Does the Potential Energy of a Rising Helium-Filled Balloon Change?" Physics Teacher 40:164-65.

Kosso, Peter. 2000. "Fundamental and Accidental Symmetries." International Studies in the Philosophy of Science 14:109-21.

Lanczos, Cornelius. 1986. The Variational Principles of Mechanics. New York: Dover.

Lange, Marc. 2007. "Laws and Meta-laws of Nature: Conservation Laws and Symmetries." Studies in History and Philosophy of Modern Physics 38:457-81.

- 2009. Laws and Lawmakers. New York: Oxford University Press.

Lederman, Leon, and Dick Teresi. 1993. The God Particle. New York: Dell.

Leroy, Bernard. 1985. "Archimedes Principle: A Simple Derivation." European Journal of Physics 6:56.

Mach, Ernst. 1960. The Science of Mechanics. La Salle, IL: Open Court.

Ne'eman, Yuval, and Yoram Kirsh. 1996. The Particle Hunters. Cambridge: Cambridge University Press.

Planck, Max. 1948. "Ansprache des Vorsitzenden Sekretars, Gehalten in der öffentlichen Sitzung zur Feier des Leibnizschen Jahrestages, 29 June 1922.” In Max Planck in Seinen Akademie-Ansprachen, 46-48. Berlin: Akademie-Verlag.

Pnueli, David, and Chaim Gutfinger. 1992. Fluid Mechanics. Cambridge: Cambridge University Press.

Rudiak, V. M. 1964. "A Proof of Archimedes' Principle.” Physics Teacher 2:293.

Salmon, Wesley C. 1989. "Four Decades of Scientific Explanation." In Scientific Explanation: Minnesota Studies in the Philosophy of Science, vol. 13, ed. Philip Kitcher and Wesley Salmon, 3-219. Minneapolis: University of Minnesota Press.

Steiner, Mark. 1978. "Mathematics, Explanation, and Scientific Knowledge.” Noûs 12:17-28.

Tarasov, Lev, and Aldina Tarasova. 1973. Questions and Problems in School Physics. Moscow: Mir.

Trefil, James. 2003. The Nature of Science: An A-Z Guide to the Laws and Principles Governing Our Universe. Boston: Houghton Mifflin.

Weinberg, Steven. 1995. The Quantum Theory of Fields. Vol. 1. Cambridge: Cambridge University Press.

Whewell, William. 1874. History of the Inductive Sciences. Vol. 1, 3rd ed. New York: Appleton.

Wigner, Eugene. 1972. "Events, Laws of Nature, and Invariance Principles." In Collected Papers, pt. A, vol. 3, 185-96. Berlin: Springer. 